\title{
Personas revisited: Extending the Use of Personas to Enhance Participatory Design
}

\author{
Judy Bowen \\ Computing and Mathematical Sciences, University of \\ Waikato, Hamilton, New Zealand \\ jbowen@waikato.ac.nz \\ Annika Hinze \\ Computing and Mathematical Sciences, University of \\ Waikato, Hamilton, New Zealand \\ hinze@waikato.ac.nz
}

\author{
Helen Petrie \\ Department of Computer Science, University of York, York, \\ United Kingdom \\ helen.petrieac.uk@york. \\ Sanjit Samaddar \\ Department of Computer Science, University of York, York, \\ United Kingdom \\ sanjit.samaddar@york.ac.uk
}

\begin{abstract}
Personas are a well-established tool in interactive system development, largely used with designers and developers. They can also be included in work with potential and actual users, particularly in eliciting user needs and concerns. We present three case studies in which personas are part of the research with potential users of interactive systems. Blind participants in research on assistive technologies often spontaneously generated personas who would benefit more from the technology than they themselves would. This was a way of being able to discuss potential needs and concerns, without admitting to having them oneself. Older participants exploring the potential of indoor drones generated personas who were older than themselves and would need the system more. Finally, personas were created to aid discussions with forestry workers about fatigue and safety issues that they might not want to admit to having themselves. From these case studies we draw recommendations for how personas can be deployed in working with potential and actual users of interactive systems in a variety of ways.
\end{abstract}

\section{CCS CONCEPTS}

- Human computer interaction (HCI); • HCI design and evaluation methods; • User models; • Interaction design; • Interaction design process and methods; • User centered design.;

\section{KEYWORDS}

Personas, user research, participatory design, blind users, older users, forestry workers

ACM Reference Format:

Judy Bowen, Helen Petrie, Annika Hinze, and Sanjit Samaddar. 2020. Personas revisited: Extending the Use of Personas to Enhance Participatory Design. In Proceedings of the 11th Nordic Conference on Human-Computer Interaction: Shaping Experiences, Shaping Society (NordiCHI '20), October

Permission to make digital or hard copies of all or part of this work for personal or classroom use is granted without fee provided that copies are not made or distributed for profit or commercial advantage and that copies bear this notice and the full citation on the first page. Copyrights for components of this work owned by others than the author(s) must be honored. Abstracting with credit is permitted. To copy otherwise, or republish, to post on servers or to redistribute to lists, requires prior specific permission and/or a fee. Request permissions from permissions@acm.org.

NordiCHI '20, October 25-29, 2020, Tallinn, Estonia

(c) 2020 Copyright held by the owner/author(s). Publication rights licensed to ACM ACM ISBN 978-1-4503-7579-5/20/10 ..\$15.00

https://doi.org/10.1145/3419249.3420135
25-29, 2020, Tallinn, Estonia. ACM, New York, NY, USA, 12 pages. https: //doi.org/10.1145/3419249.3420135

\section{INTRODUCTION}

The use of personas is a well-established and researched approach in interactive system design. They are typically employed by designers as a way of introducing archetypal users and their needs and aspirations into early phases of the design process. Personas were originally intended to enable designers to focus on people who were not themselves (so designing for actual users rather than their own needs) and so were intended to be representative of the target user groups or of a specific user within that group. Within the rich body of literature on personas there are many opinions on the correct ways of developing personas and validating them (e.g. see Section 2) as well as how and when they should be used by various members of the design and development teams.

However, what is seldom discussed in the literature is the use of personas with potential or actual users of interactive systems, for example within a participatory design process (for some exceptions, see Section 2). Participatory design is a user-centred design approach which is intended to allow the intended users of the systems under development to be deeply included in the design and development process. This ensures that users' needs and aspirations, alongside their opinions and attitudes to proposed technologies can be understood and incorporated by the design team. In this way, the participants meaningfully contribute to the end product. However, there can be problems with participatory design approaches. They assume that designers always have access to a group of potential users who are both willing and able to take part in such a design process. They also require that the users can articulate their needs and aspirations clearly and confidently and that they are comfortable to do so in discussions with their peers and designers. As technology becomes more ubiquitous and aims to tackle social and health challenges these discussions may be increasingly personal and focussed on aspects of users' lives and personalities that are less easy for them to discuss.

In this paper we show that using personas with potential or actual users in the participatory design process can provide a mechanism for them to express opinions and emotions that they may not be comfortable sharing if asked directly about their own opinions. We propose using the personas along with the participant group as an additional member of that group, rather than a more traditional 
approach of using a persona to replace actual participants. In addition, we demonstrate that the use of personas in this way may assist with focussing participants on particular aspects of a design that would otherwise not seem important to them. As such the personas can act as a shared basis for communication as described by Grudin and Pruitt [20,37] but between users and designers rather than designers and developers or other stakeholders. We present three case studies in which the use of personas with participants has been used successfully. In each case study, the personas were created and used in different ways, as proxy personas and emergent personas. In this way participants could adopt the tactic of "asking for a friend" (where personal or embarrassing questions are attributed to someone else) by discussing the persona and attributing them with difficult feelings or revelations. We describe each case study along with a rationale for how and why the personas were introduced and discuss the effect the personas had on the particular design process. We then present a discussion on our findings across these three case studies and consider the implications and what can be learned from these findings. Finally, we present concluding remarks and discuss future work

\section{BACKGROUND AND RELATED WORK}

Personas were originally proposed in the late 1990s by Cooper [11] as an effective and efficient way of engaging designers and developers with the characteristics and needs of their users. For Cooper, personas are user models, composite archetypes based on behaviour patterns uncovered during research with users that might include ethnographic observation, contextual inquiry, interviews, focus groups, usability testing, card sorting and even task analysis (all these methods are described in his most recent book [12]). The information from this user research is then condensed into a number of fictional people who illustrate key needs and aspirations, behaviour patterns and goals (see Figure 1, Section 5.1, as an example). As Cooper noted: "it is not enough to whip up a couple of user profiles based on stereotypes and generalisations ... considerable rigour and finesse must be applied during the process of identifying the significant and meaningful patterns in user behaviour and determining how these behaviours translate into archetypes" [12, p62].

Since Cooper's initial proposal, personas have become very popular, widely used in practice and studied in research. In the research world, personas received an initial boost from Adlin, Grudin and Pruitt $[1,20,37]$ whose widely cited papers and books promoted personas as a powerful complement to other usability methods, although they somewhat changed their use: "to communicate a broader range of information to more people: to designers, developers, testers, writers, managers, marketers, and others" [37], p1]. Interestingly, they do not mention using personas in work with potential or actual users of systems. Personas have also not been without their critics. Chapman and Milham [10] argued that personas cannot be adequately verified or falsified and that the effectiveness of personas had not even been established.

However, since the critiques of validity and effectiveness, research has addressed both these issues. On the issue of validity, a number of approaches have been proposed to generate personas automatically or semi-automatically from large datasets about users, to overcome the biases arising from manual construction based on small samples of data. For example, McGinn and Kotamraju [26] conducted a survey of 1300 potential customers and conducted factor analysis on the resulting data to create what they argued were statistically valid personas. Jung and colleagues [23, 24] conducted analyses of 30 million views of a particular YouTube channel to create their personas. Of course, not all researchers or practitioners have access or the resources to analyse these amounts of data. Faily and Flechais $[15,16]$ proposed a much more fine-grained approach, suitable for smaller datasets, using grounded theory analysis of empirical data to show the validity of personas. Finally, Salminen and colleagues [39] also proposed a different approach of using a Persona Perception Scale to ask experts to complete about proposed personas. However, it is not clear how experts are supposed to relate their responses on the scale to the empirical data used to generate the personas.

On the issue of effectiveness, Chang, Lim and Stoltermann [9] conducted a small study investigating a design exercise which compared two two-person teams, both given the same brief, but one included a persona and the other did not. The analysis of discussions of the two teams showed that even the team which were not given a persona used an "unspoken persona", that is they imagined a typical user as they were designing the system. Long [25] took the idea of a design exercise further, comparing 9 student project teams: three teams had a design brief; three had a design brief with a persona and a text-based scenario; and three had a design brief with a persona and a storyboard scenario. Marks for the projects and heuristic evaluations of the project products showed that both groups of projects with personas scored higher marks and achieved better usability ratings than the brief only projects, and that the projects with a storyboard scenario scored better than a text-based scenario.

Another series of studies in a number of different countries have interviewed practitioners to investigate the effectiveness of personas. Matthews, Judge and Whittaker [28] interviewed 12 practitioners in the USA and found three were very positive about personas (persona champions), six were moderately positive and five were negative (persona pessimists). In addition, they found that personas were not generally used in design, but for communication with non-designers and non-UX practitioners about users and user-centred design issues. Nielsen and Hansen [32] interviewed 28 Danish practitioners and most described their work with personas as satisfactory or very satisfactory, although that was often after several failed attempts at using them. These practitioners reported using personas in many ways during the design process, but none reported using them with potential or actual users. Finally, Marsden and Haag [27] interviewed seven German practitioners and found that personas run the risk of reinforcing existing stereotypes, with agendas such as inclusive design, anti-discrimination or sustainability ruled out.

A further series of studies investigated how different professionals who use personas react to different aspects of the personas and whether their perceptions of their users are accurate. For example, Nielsen, Jung, An, Salminen, Kwak and Jansen [31] compared media professionals views of their audience with personas automatically generated from audience Facebook accounts. They found that the professionals were not had particularly accurate in their 
understanding of their audience. Salminen, Nielsen, Jung, An, Kwak and Jansen [40] then used the automatically generated personas to study whether different types of photos in a persona affected their interpretation. They found that more contextual photos (i.e. of the person in different situations and with different other individuals) significantly improved the information professionals derived from a persona.

The concept of personas has also evolved in many creative and interesting ways. For example, Djajadiningrat, Gaver and Frens [13] turned the idea of an archetypal persona on its head by developing extreme personas to explore emotional reactions and cultural issues. The personas included a drug dealer, the Pope and a hedonistic 20 year old woman. Nieters, Ivaturi and Ahmed [33] found that personas were not engaging relevant stakeholders in their organization, so instead of creating archetypal individuals, they creating characters based on action figures with "super hero" qualities, such as "Keep-his-cool Kent", whose tagline was "My profile says 'specialist' but 'superman' is more like it". In a similar vein, Anvari et al. [2] added personality to their personas and found this did affect the way student designers worked with them. Finally, Quintana et al. [38] proposed a "persona party" to engage a multidisciplinary design group who are creating a massive online open course (MOOC).

As noted, personas are almost always seen as a tool for the development team, to help designers and developers understand the needs and aspirations of the users. Only a small number of strands of research could be found that have used personas with potential or actual users of systems as part of the design process.

Hisham [21] used a persona when eliciting requirements from older users in Malaysia for an email application. She created a persona of an older woman who wants to use email to communicate with her adult children who live abroad, but has a range of difficulties in using both the hardware and software to do so. She used the persona in a focus group with eight older Malaysians, verbally explaining the persona to the group and asking them to question and discuss it. This proved a very effective procedure, as the focus group was due to last 45 - 60 minutes, but discussion continued for two hours. Hisham attributes the success to four factors. Firstly, she felt that the use of the persona brought focus to the discussion, meaning that participants concentrated on the different issues raised in the persona, rather than the discussion going off topic. Secondly, the persona encouraged contributions from participants who were not users of the technology or not confident about the technology, as they could talk about the persona and her problems. Thirdly, the persona greatly stimulated discussion, as numerous participants used the persona as a bridge to their own experiences and problems. Finally, the persona built interest in the topic, with participants asking for more information and wanting to learn more about the email application.

Nielsen [30] also used personas in two focus group situations, one with cafeteria managers and one with mothers of children who engage in sports. The aim in both these situations does not appear to have been to facilitate discussion of difficult topics, but to help the participants gain insight into the needs and wants of other stakeholders: cafeteria customers in the case of the managers, and parents, sports trainers and children, in the case of the mothers.
The personas proved a useful starting point for role playing and discussions of different possible scenarios and technological solutions to problems in each case.

Cabrero and colleagues $[7,8]$ investigated personas with potential technology users in several rural settings in Namibia, particularly because they felt that personas are potentially a very WEIRD (i.e. Western, Educated, Industrialized, Rich and Democratic) way of representing user needs and aspirations and they wanted to investigate whether laypeople (i.e. non-HCI/UX experts) could use personas in a co-design technology development process. They worked with several different rural groups of participants in Namibia and found that people in those cultures did indeed initially find the concept of a persona very alien. However, through explanations, discussions and negotiations with the researchers and interpreters, participants were able to create personas reflecting archetypal individuals in their societies, although there were suggestions that these participants had very different ways of conceptualising a persona.

Finally, Bourazeri, Stumpf and colleagues [5, 29] used personas with potential technology users who have dementia and Parkinson's disease. Their approach is the most clearly structured of those found in the literature. They held a series of four workshops over a period of six months. In the first workshop the researchers and co-designers with either Parkinson's or dementia created several personas. In the second workshop they then revisited them to refine them and to reflect on the personas' potential use of technology. In a third workshop they co-designed a low fidelity prototype and in the fourth workshop they evaluated the prototype. Their research shows that working with users with disabilities and chronic health conditions can be very time consuming and demanding (e.g. several participants who were involved in the early workshops were unable to continue due to health issues or loss of interest, and different participants had to be replaced), but also very rewarding and extremely beneficial for the resulting designs.

Having presented a brief history of the development of personas in $\mathrm{HCI}$ and their limited previous use with potential or actual users in the design process, we now turn to our three cases studies of different ways we have used personas in participatory design projects.

\section{CASE STUDY 1: EARLY EXPERIENCES WITH BLIND AND VISUALLY IMPAIRED PEOPLE}

Over a period of years, we have worked on a number of projects with people who are blind or severely visually impaired. These have particularly included projects about how blind and visually impaired people (VIPs) navigate the outdoor environment [e.g. 17, 22, 34, 42] and use the Web [e.g. 36, 41] and other interactive technologies [e.g. 35]. We were very conscious that it is usually not possible to work with a fully representative sample of people from these user groups, most research projects recruit relatively small samples of self-selecting people and these are usually those who are most confident in their skills, be that in using technology or in outdoor mobility. The use of personas helped overcome these problems.

For example, in several outdoor navigation projects we were only able to recruit blind people who were confident enough to travel to 
the university, albeit frequently assisted by the researchers. However, the projects also aimed to help blind and visually impaired people who were not confident enough to travel by themselves. We began to very informally create personas of different types of blind and visually impaired travellers to see whether our participants identified with them and could give us insight into the needs of the potential users we were having difficulty reaching, we call these "emergent" personas, as they were not formally planned before the design sessions and presented as personas to the participants, but introduced informally in discussions. For example, we would ask participants about their own needs and reactions to navigational support, but also ask them to think about a blind person who might be less confident themselves about travelling to an unknown location by themselves than they were and a blind person who might currently not travel independently at all. We found that some, but not all, of our participants, were comfortable with engaging in these speculations about people with characteristics different from themselves. This often depended on the life experiences of the individuals. Some blind and visually impaired people know few other people who are blind or visually impaired, so this was simply not something they felt comfortable speculating about. However, many people in the UK who are visually impaired from birth or from a very young age have been educated in schools for blind and visually impaired students, so they do know a range of blind and visually impaired people, and were quite comfortable with these discussions. It was hard for us to validate whether the insights from our informants were accurate in helping us understand the needs of less confident blind and visually impaired people. However, we did have several "eureka" moments when participants who had said they would not travel alone to an unknown environment (as opposed to a known environment, this being a very important distinction for blind and visually impaired people) said they would be confident to do so using our system. So, we believed we could assist blind and visually impaired people in moving along a continuum from dependence on others for mobility to greater independence in mobility, which might therefore also be valid at lower levels of independence.

When we moved on to study digital accessibility, particularly web accessibility, we encountered an interesting variation of this situation. Again, we found it very difficult to recruit participants with less than excellent digital skills, in spite of considerable efforts to do so. Quite possibly, those who were less confident in their skills did not want to volunteer to be in research projects, as they feel it would expose their lack of expertise. Interestingly when we did recruit people with lower levels of skills, they often found the sessions very frustrating, as they would struggle with the technology. This was sometimes interesting for the researchers, but generally did not reveal much of use to the research. But more importantly, we did not want to have people come and do research with us and only have a frustrating experience. However, we found that the participants with high digital skills were often very good and quite willing to engage with emergent personas of less skilled individuals. In these sessions we often did not have to propose a persona ourselves, the participant would come up with different persona themselves, for example saying things such as "not many screenreader users would know how to use this feature ..." or "typically people using [xxx] would do it that way, but once you know it's quicker to do it this way ...". So the participants were generating the personas and explaining them to the researchers. We would often then encourage further exploration of different types of personas.

One reason that the digital skills situation seemed to come more naturally and more fluently to our participants may have been that many of them taught digital skills to other blind and visually impaired people, whether formally or informally. So, they had much more explicit knowledge about what people with different skill levels would be likely to know or do. However, there was an interesting, more psychologically subtle difference between the dialogues around mobility and those around digital skills. Those who had good digital skills were proud of these and keen to show how good they were with technology. Whereas with the mobility issue, they did not want to denigrate other people's lesser skills and confidence.

Overall, in working with blind and visually impaired people, we have found that informal, emergent personas were very useful as a tool to extend discussions about user needs and aspirations, although they did not work with all participants in all situations.

\section{CASE STUDY 2: ROBOTIC AGENTS FOR OLDER PEOPLE}

\subsection{The GUFO Project}

The GUFO project investigated the use of an indoor drone to assist older people living independently in their own homes. The proposed system consists of a small drone, a smartwatch, and AIbased software. A key function of the system is fall detection, in which the system will inform a carer, relative or the emergency services if the older user has a fall and requires attention. The smartwatch will track heart rate, balance and acceleration to help determine whether a fall has taken place (as opposed to the user simply falling asleep on the sofa). The drone will then autonomously fly around each room of the home to locate the user using image processing and AI, ask the user whether they need assistance and depending on the response, seek appropriate help. The drone will also be able to do regular checks of the home to ensure that all is in order, for example taps have not been left running, the cooking stove not left on, windows or doors left inappropriately open and so on. The smartwatch will also give access to a range of features such as reminders to take medications, finding household objects such as glasses and TV remotes, and contacting family members.

Much of the technology used in the GUFO system would be relatively new for the target age group, people currently aged 65 to 85 years. Therefore, extensive user studies were needed to investigate the attitudes and preferences of the target users in relation to the technologies involved. The drone and the smartwatch were the focus of three studies we conducted with potential users. In the course of the studies, a number of different drones were presented to the participants and they were interviewed about their opinions on the drone and having the GUFO system in their homes. Following that, the user interface for the smartwatch functions was developed using participatory design processes. The design was iteratively developed with each user study 
providing further opportunity for comment and design with the participants.

In the first user study, we experienced difficulties in eliciting appropriate information from our participants for several reasons. Firstly, the majority of participants were recruited through an online community forum, and were asked to come to the Computer Science Department at the University of York. This resulted in a self-selecting sample of digitally skilled and confident participants who were used to using laptops or tablets and were capable of travelling to the department. While all participants were over 65 years of age (range: $65-82$ ), most were considered 'young-old', being between 65 and 74 [3]. These participants would not be users of the system in the immediate future, although they might well be in a few years' time, and had not experienced age-related falls yet, so were not able to always see themselves as users of the system. In addition, digitally skilled, they were familiar with the idea of robots and drones even if they had never seen them in person.

Secondly, and most importantly, we noticed participants were hesitant to admit that they needed help in living independently. Often a participant would say something to the effect: "I don't have any difficulties, but I know others who do" (P05). In addition, when reacting to the demonstrations of the drones, many participants seemed to be surprised by the noise and wind it created, but when asked would emphasise that they had no issues with it, when we suspected they did have issues, but did not want to voice them.. When answering questions participants frequently queried: "Should I answer this as I am now or for me in 5-10 years' time?" (P11). Thirdly, when discussing the design for the smartwatch, we often observed that participants had to squint to see the small screen or repeatedly tap the screen for it to register an input. But when we suggested improvements to the design to help with these issues, participants often dismissed them, stating the problem was they were just not used to the new screen or that they were not able to see it because of the sunlight in the room. In the latter instance, participants may well not have understood that touch screens do not work as well for older fingers which are drier than young ones; but in the first instance, they undoubtedly realised that they could not see the small screen due to diminishing visual acuity. Participants spontaneously started referring to older friends and family when answering questions, going as far as rating Likert items twice, once as themselves and once as their older family member. And comments such as "Oh my mum would have real difficulty using that screen" (P02) were frequent. While all the participants stated that they greatly appreciated the need for the GUFO system, they were clearly hesitant to admit needing it in their own homes and were hesitant to suggest improvements to the designs, perhaps in fear of coming across "old" or "stupid".

These experiences led us to use emergent personas in the two subsequent studies in a more considered manner than we had in the work with blind and visually impaired participants. Building on the spontaneous persona generation by the participants in the first GUFO study, these personas were friends or family members who were older and may be homebound, have physical disabilities or generally not be as good with technology as the participant. The development and use of these personas are presented below.

\subsection{Participatory Design Process in the GUFO Project}

4.2.1 Developing the GUFO Personas. From the second user study onwards, we developed and used emergent personas with our older participants. The personas were focused on three areas of characteristics: age, use and expertise with technology, and physical or mental disabilities. The age of the personas was always kept ambiguous and referred to as "someone slightly older than you". This was done as to keep the personas fluid between participants and also allow for the participant to come up with their own perception of what 'older than them' meant. 95\% of our participants considered themselves frequent users of smart devices and therefore the persona was developed as someone who may not use a tablet or smartphone and rarely used a computer. Finally, the personas were developed to have some disabilities or difficulties related to old age. For the GUFO project this was either someone who was chair bound or someone who had dementia.

These characteristics were used to introduce and describe the persona in an informal way to participants. They were not introduced explicitly as a persona, but the researcher suggested the participant should answer for their own needs and wishes and in relation to a person like themselves but with these characteristics. The rest of the more humanising characteristics such as goals, motivations, feeling and behaviours were created on a more ad-hoc basis. As noticed from the first user study, participants would usually mention older friends and family members. In using their own descriptions and asking about these older friends or relatives, we would flesh out the persona and use it to establish rapport with the participants but also build on the personas we had in mind. The persona would be able to take on the role of their friend and participants could bring in characteristics or behaviours of people they know while also being able to comment more freely on how the persona might not be as comfortable with the smartwatch or might be afraid of the drone.

As with the participatory design process for the smartwatch interface, the personas were also elaborated on during each participant session. If a participant responded more to a persona with traits of an older relative, that persona was developed with that participant. If a participant responded more to a persona with disabilities, that persona was developed with that participant. The personas were not designed directly with the participants but rather built and added to by the researchers in repeated interactions with the participants. In follow-up sessions, the persona would be introduced by saying, "let us think about someone like your older friend again. ..". This process is detailed further in the next section.

4.2.2 Using the GUFO Personas. The personas were introduced to participants at the start of a co-design session. Instead of providing a template or physical representation of a persona, the personas were introduced as older friends or relatives. Participants were told that they should think about a person who is slightly older than them, probably not as much of an expert in using technology and might have some physical disabilities which makes them less mobile. We found that giving an ambiguous description of a persona worked very well with our participants. The participants frequently noted that they could easily think of such a person and could empathise that they themselves might face the same problems in the future. 
For example, one participant noted: "I have a friend who can't walk her dog anymore and had to give it up. I don't have that problem but never say never!" (P06).

In the second user study, when given a demonstration of a drone, very few participants initially expressed any concerns, as in the first user study. However, with minimal prompting about the persona, they would produce comments such as: "Now if I think of my older friend, I can see her being very afraid of this loud buzzing machine" (P14). The benefits of using the personas was even more evident when discussing the smartwatch. Participants would struggle with some aspect of the smartwatch interaction but not comment on why they were struggling. Many participants started producing comments such as: "My friend doesn't like using touch screens because they don't work with her fingers" (P06) or "My mother cannot use a smartphone, her hand shakes sometimes" (P01). These comments unlocked opportunities for further discussion of the problems and possible improvements to the design. The participants could add how they think the design could be improved for the personas without having to admit that it would also make the interaction easier for them. For example, one participant commented: "Now you see, if my older friend couldn't use the touchscreen she would get very angry and start tapping the screen multiple times! Is there something to manage that?" (P06).

The use of the personas showed us a clear advantage of providing a realistic persona who could shoulder the blame for being older and needing help rather than requiring that the participant discuss their own difficulties. In addition, in some cases, having cared for relatives with dementia or having frequently interacted with old neighbours and friends, participants provided rich detail about their experiences with real people while also maintaining anonymity and respect for these individuals. One participant noted: "I don't want to embarrass my mum and say she is stupid, but thinking about a person like her who has never used anything but a TV remote, they would have no idea how to work this contraption" (P22).

With the success and amount of information elicited using the personas, we continued to develop the personas and use them in a further user study in the GUFO Project and in other projects. As the personas were individually developed to some extent for each participant, a record of the characteristics of the persona was kept, so that if that participant took part in subsequent sessions, we could return to their personas. The next section explores how these personas were used and adapted for a different research project with participants who had taken part in the GUFO and new participants.

4.2.3 Re-use of the GUFO Personas. To explore the use of the personas further, we used them again in another research project. This project investigated the preferences of older people when interacting with life-like avatars. We invited participants from the GUFO study and in addition recruited further participants.

For the participants who had helped with the GUFO study, the personas were introduced by reminding them of the person they had talked about previously, particularly an older friend or family member. In this case, most participants felt that the older person might be too old for the avatars, having already stated that they themselves would have no problem interacting with the avatars. It was difficult to determine if this was them using the persona to voice their own concerns or whether they genuinely thought that someone older than them would not use the avatars. Some participants found it easier to imagine their chosen person using the avatar even if the person was not a part of their lives anymore. For example, one participant commented: "Oh, I've fallen out with her. I don't know what she is up to (P16). But yes, I think someone like her would find it a bit weird to talk to a virtual human”. For new participants, the personas were introduced as described for the GUFO studies. Even without a previous discussion of a friend or family member, all participants seemed to voice their thoughts using the personas. Often, defaulting into a familiar pattern of the comment by one participant that: "I don't see any problem with this guy [male avatar on screen], but I know someone who would...”.

This demonstrated, once again, the advantage of using a persona in studies with older people and how personas can be used as a secondary participant when a participant does not want to discuss their own needs, aspirations and problems. Further work needs to investigate the limits to how and when an emergent persona can be used in participatory design with older people, but the GUFO and avatar user studies have shown that there is a useful place for personas in user studies with older people.

\section{CASE STUDY 3: THE HAKITURI PROJECT}

This section introduces the Hakituri project (Section 5.1) and outlines how personas were used in the project design along with the actual participants during participatory design activities (Section $5.2)$.

\subsection{User Studies in the Hakituri Project}

The Hakituri project aims to develop wearable technology for forestry workers with the goal of identifying and reporting worker fatigue to reduce accident rates. For the past six years the research team have been working with forestry teams of varying sizes for a number of different studies $[6,19]$. In the early parts of the research project, the studies typically involved working with small teams (3 - 8 workers) testing out different types of wearable technology that measured activity levels and personal metrics such as heart rate. These studies aimed to investigate the suitability, accuracy and acceptability of the different types of wearable devices. From these early studies we identified participant traits that were evident across all of the teams, and which influenced our later work. We were also able to identify potential problems that would impact later studies based on worker buy-in and how they felt about being monitored by wearable devices during their working day.

In each case, the study teams were identified through primary contacts in forestry organisations. Once a company or team agreed to take part (typically arranged by the manager of the crew) then participants were identified by their managers and recruited into the research project. As such the individuals did not directly volunteer for the project, but all gave their consent to take part. This is one of the factors that influenced the willingness of the workers to fully engage with the process and which needed to be considered in our subsequent studies. Another factor was the misunderstanding about what the wearable technology was able to measure and identify. Examining the data of one study showed that one of the participants would have no heart rate recorded for a period of about ten minutes at roughly the same time each afternoon. Our hypothesis (based on 
some known behaviours of the workers) was that he was removing the wearable device before going for an illicit cigarette break as he thought the device would be able to report what he was doing.

These early studies also identified that disruptions to the study and validity of the results could occur due to these factors. For example, workers would interact with the provided technology in unexpected ways. These could be due one of a number of reasons: the fact that they were technology novices unfamiliar with aspects of use; poor usability of the technology; reluctance to engage in some parts of the study process; reluctance to commit personal time to the study.

More recently, we undertook participatory design workshops with targeted groups of forestry workers. The studies we discuss in this paper were undertaken with groups of Maori forestry workers. Maori are the indigenous population of New Zealand, and represent a large cohort within the forestry industry. The workshops were designed to find out forestry worker attitudes to using wearable technology to prevent fatigue-related accidents. The wearable technology would measure things like heart-rate variability and galvanic skin response to identify fatigue. Data that was collected would be used to warn workers that they were fatigued or dehydrated and at increased risk of accident. Data would also be collated and reported back so that workers, their family, and supervisors could observe overall trends over time. Our workshops consisted of three participatory design sessions each with two groups of forestry workers (so six in total). Workers undertook activities designed to explore their feelings around health and wellbeing, wearable technology in the workplace, the use of personal data and how it is shared. Group 1 consisted of 20-25 silviculture (tree planting) trainees (not all attended all three sessions) who were undertaking a programme with a local forestry contracting organisation as part of a Government initiative. The majority of Group 1 participants were in the younger age range (under 30 years) and the group had the potential to be quite volatile due to pre-existing conflicts and external factors. Supervisors and office staff were also present, but they primarily took the role of helping to facilitate the workshop sessions rather than contributing directly. Group 2 consisted of workers and family members from a family owned and run forestry contracting company. This group comprised mostly older participants (over 30 years) along with elders (grandparents who had been involved in setting up the company) and other family members such as wives, partners and children (several pre-school age). This created a very different group dynamic from Group 1 and also meant that there was already a lot of shared personal information among the participants.

Employee demographics in forestry play a large part in determining how participants engage in various parts of our research. Forestry is a physically demanding job with a high staff turnover (of the trainees in our first group of participants only $50 \%$ are still in the industry 10 months later). Many employees cite the long working hours and low rates of pay as reasons for leaving forestry employment. Employees are primarily male (96\%) and below the age of $50(65 \%)$ with $5 \%$ of employees being below the age of 20 [14]. The work culture means that workers themselves can often be described as having a 'staunch kiwi male' attitude (which might also be described as macho), which can lead to a minimising of potential risks and a desire to not appear weak in front of workmates. For example, some of the comments from participants in Group 1 on the use of wearable technology to identify fatigue was particularly pertinent:

"You think you're bulletproof in the forest"

"Can we add one big no, no? ......That it's gonna make us pussies."

This attitude was particularly relevant for our participatory design workshops where we asked the workers to discuss aspects relating to health, fatigue and managing personal safety in the workplace. We determined that it would be useful to provide a way for the workers to share concern and opinions about their understanding of health, fatigue and work safety in a way that would potentially be more comfortable for them in a setting where they were interacting with their peers. This led to our decision to create personas that we introduced into the participatory design sessions.

\subsection{Participatory Design Process in the Hakituri Project}

5.2.1 Developing the Hakituri Personas. In our participatory design we wished to use the personas to extend the participant group (i.e., the personas become part of the group) and so it was important that they 'fit in' by being as similar to the participants as possible. We therefore developed the personas by using attributes of earlier participants in our forestry user studies, known demographics of forestry workers in New Zealand and characteristics of people we had met during the previous six years of the project. The actual persona descriptions used are 'lightweight' in that they provide only brief descriptions which focus on two or three personal attributes.

Six personas were developed, all were male and their ages ranged from 16 - 52. These demographics were chosen as reflecting typical participants in the project (and matched exactly the demographics of the participant teams they were used with). For five of the six personas, names and/or photos indicated a Maori cultural background. Two of the personas were related to each other, to again reflect the fact that this is a common occurrence in forestry teams (as discussed above). The personas had a range of years of experience in forestry (from novice to more than 15 years) and different roles within the industry, including one in a supervisory role.

While it is typical to consider users in a design process based on their technical skills, for our personas it was more important for us to consider forestry experience and roles to ensure that all of our real participants could feel represented by at least one of the personas. So, while the younger workers might express themselves through the Jordan persona (16 years old and a novice), the older team members and those in supervisory roles could attribute their feelings and comments to Tahuri (51 year old supervisor).

The personas were assigned with hobbies that reflected both common activities for our participants (playing rugby league, fishing) as well as age-specific traits (playing video games or watching online material for the younger participants), Each was also given a 'humanising' factor that was intended to make them more realistic to the participants e.g. one persona is divorced and trying to get a better place to live so that his children can visit more, one is trying to save enough money to get a car etc. One of the personas (Jordan) was given the humanising factor of being asthmatic, this led to some unexpected consequences in the design sessions which 


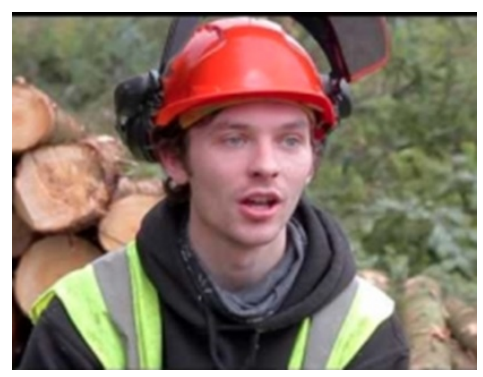

Jordan Henare, age 16 Jordan's father and three uncles all work in forestry and he was keen to join them as soon as he could leave school. His cousin Kahurangi works with the same crew and has been helping him to get used to the job. Jordan's biggest challenge is getting up early in the morning for work, he likes to watch stuff online during the night and isn't very good at waking up when his alarm goes off. Sometimes he stays with Kahurangi so he can go to work with him and be on time. Jordan has asthma but he doesn't always remember to use his inhaler.

Figure 1: Persona from the Hakituri Project.

we discuss later. Photos were selected from stock images online of forestry workers or manual workers and were chosen based on how well their appearance matched the required demographics. An example of one of the personas is given in Figure 1

\subsection{Using the Hakituri Personas}

When setting up the participatory design sessions we needed to be mindful of the male-dominated culture and consider how this might impact information we wanted to elicit.

In the first session the focus was on personal health and wellbeing, as well as attitudes to monitoring technology in the workplace. For this we relied on the participants to reflect on their own attitudes and provide personal responses. Group 1 (the Younger group) provided more generalised answers to personal health questions (or occasionally referred to people who were absent). Their technology envisionments were around making things fast (rocket boots and drones) rather than making themselves safer and their concerns were not around who might have access to their personal data, but rather that the research team might steal their ideas to make lots of money. Because Group 2 included family members of the workers, this had the effect of tempering the conversation and also providing more 'real' input. For example, the wife of one of the workers was prepared to share her husband's personal health status as a way of describing what was important to her and why.

The personas were introduced in the second of three sessions with each group. The participants were divided into smaller groups (3- 5 participants per group) and each was given one of the persona profiles and told that they should consider this person as a member of their work crew. We did not describe the process of creating the personas or give any background on the use of personas within technology design.

Although the participants were told that the personas were not actual people, there was some discussion in one of the sub-groups as to whether or not their persona was someone they knew or was someone's cousin. While we reassured them that this was not the case, we also took this as affirmation that the personas were believable and representative of the group to the point where they accepted them as real people.

One of the goals of using personas was to create a distance between the workers and their personal feelings or behaviours. By allowing them to attribute these to 'someone else' (the persona) we hoped it would enable them to speak more freely. Also, for the younger group where there might be potential tensions if they felt they were being singled out by others in the group, it was a way of defusing this. Interestingly in the Younger Group we still saw evidence of this, but it was directed at one of the personas. 'Jordan' (persona in Figure 1) was identified by the group as 'weak' (his persona description stated that he had asthma) and in the data visualisation examples we presented his statistics were the worst in the group. Comments around this were that he wouldn't last in the job and would quit or be fired because he wasn't up to it:

"He must be a slacker"

"He won't be making money that's for sure"

"Sucks to be Jordan"

In the second session the types of questions the groups were asked to focus on included describing how their persona might behave under certain circumstances as an indication of how they themselves might behave, e.g. "What if Jordan's technology indicates he is tired, but he only has one more box to finish before knock off, what do you think he would do?". In addition, we wanted to elicit how they might support each other in different circumstances, e.g. "What if you were notified about Jordan's condition when he did not stop and rest, what would you do?". In the third session we presented different data presentations for the aggregated data and asked the participants to discuss what they would be comfortable with sharing and who should have access to the data. The sample data presented was based on the personas, so for example in an overview of the work team, Jordan (who was already identified as being an under-performer) had poor statistics. We wanted to know how the participants would feel if their data were on display in this manner and so again focused this on the persona, e.g. "So do you think Jordan would feel. . .how would you feel if you were Jordan?". However, for the Younger Group this did not necessarily lead to disclosure of personal feelings, but rather was responded to in a more joking fashion (e.g. "Tired" was one response to the question about how they thought Jordan would feel).

\subsection{Reflections on the Use of Personas in the Hakituri Project}

Using the personas in this way in the Hakituri Project has enabled us to gain some insight into how the workers perceive admissions of tiredness or illness and enabled the workers to be direct in their 
comments without causing tension among the participant group. However, a side effect of Jordan's asthma was that for the group using this persona this became the focus of their design envisionments.

One of the activities involved the participant sub-groups answering the question: "If the wearable tech identified that you are tired, how should it notify you?" They were asked to consider the persona and think what would be helpful for them, but Jordan's asthma became the focus for some of the participants who were suggesting things such as a "hydration pack with built in asthma pump" or a watch which had numbers representing different emergency conditions, like "number 3 for asthma attack". A discussion also ensued between a couple of participants about who else would know that Jordan had asthma and who was responsible for making sure he had an inhaler with him at work.

The type of use we adopted for personas can be seen as an extension to what had been observed in the projects with the blind and older participant groups. Those participants would create proxies (an elderly relative, someone they know) to project their own needs onto when they did not want to acknowledge or disclose personal aspects that might make them feel vulnerable ("I wouldn't need this, but it might help person X. ...). We hoped that similarly by providing such proxies to the forestry workers it might assist with disclosure of aspects that they would otherwise not reveal.

Although the personas were designed and used in participatory design sessions to elicit information from the wider group of participants, their value in the design process does not end there. The addition of more information about the participants (based on attributed feelings and comments) means that they have morphed into more traditional data-driven personas and been fleshed out during the participatory design process. As such there is value in continuing to use them as we progress various parts of the design process. For example, when the design of data feedback interfaces begins, we have a set of personas with opinions about aspects of the data and how it is shared. Designing for these personas will provide all of the usual benefits provided by such persona use and will be enhanced by the fact that their characteristics relating to the design are well grounded in our real user population.

\section{DISCUSSION}

Each of the three case studies we have presented targeted different types of users. In addition, the participatory design processes reported were used for different purposes. In the GUFO project participants were introduced to technology such as drones and smartwatches as an assistive technology for older people with the aim of understanding how acceptable and useable it would be for the target group. The projects for blind and visually impaired users considered navigation in outdoor environments as well as web accessibility and sought to gather detailed user requirements and understand barriers to use. In the Hakituri project, the aim was to understand how participants felt about the use of wearable technology in the workplace and how it might impact their safety and well-being. While each of these case studies were quite different, they all required participants to reveal vulnerabilities or discuss personal aspects of their lives, health and well-being which may be challenging in a participatory design environment. For this reason, we believe they were all suitable case studies for the exploration of the use of personas in the manners we have suggested.

\subsection{Persona Design Considerations}

From our three cases studies, we made a number of observations that may help in creating more suitable personas for use with participants. For the GUFO project, the personas needed to be similar to the participants but not quite them. It was more like themselves in the future (when they might be more incapacitated by age-related issues or less capable). In the projects with blind and visually impaired participants, the personas were typically someone who had similar limitations to the participant, but less capability and confidence, particularly in relation to independent travel and use of technology. In the Hakituri project, it was important that the personas were seen to be just like the participants so that they could easily imagine working alongside these people. In each of these cases the closeness of personas to actual participants meant that we did not need to create detailed back stories for the personas, as the participants could immediately fill these in themselves from the people they know or the people they were imagining. Thus, in using personas with participants, they are rather different from those which are typically used with designers, developers and other stakeholders. In those cases, providing context and back stories is very important, to help understand the users.

We also discovered that it is important to take care to avoid personal attributes that can become unnecessary distractions (e.g. Jordan's asthma). Characteristics need to be balanced, and their use needs to be guided such that a particularly noteworthy characteristic does not overshadow the other elements of the persona. However, conversely in some situations having a persona who the participants feel do not properly represent them can also be useful as it can be a starting point for conversations, somewhat like the approach of Djajadiningrat et al. [13] or provide the equivalent of a negative persona which the participants can reason about (in the manner of Hisham [21]).

\subsection{Creating Personas}

Figure 2 compares the creation of personas in the different case studies and previous research with used personas with participants (see Section 2) with respect to who is generating the personas, the participants themselves or the researchers, and whether the process is one of explicit co-creation or whether the personas emerge from the interactions between researchers and participants. We note that while traditional approaches describe explicit co-creation of personas, in both case studies 1 and 2, personas emerged from the interactions between researchers and participants. Within the same design phase (see Figure 3), these personas were then used as part of later user-centred design activities and even other projects.

It is interesting to note that just as Chang et al. [9] observed how an "unspoken persona" emerges in design processes even when personas have not been directly introduced, we observed a similar phenomenon with some of our participants. Both the blind and visually impaired participants and the GUFO participants initially created personas unprompted as a way of expressing user needs that were perhaps their own, or ones they could imagine others in their user group having. 


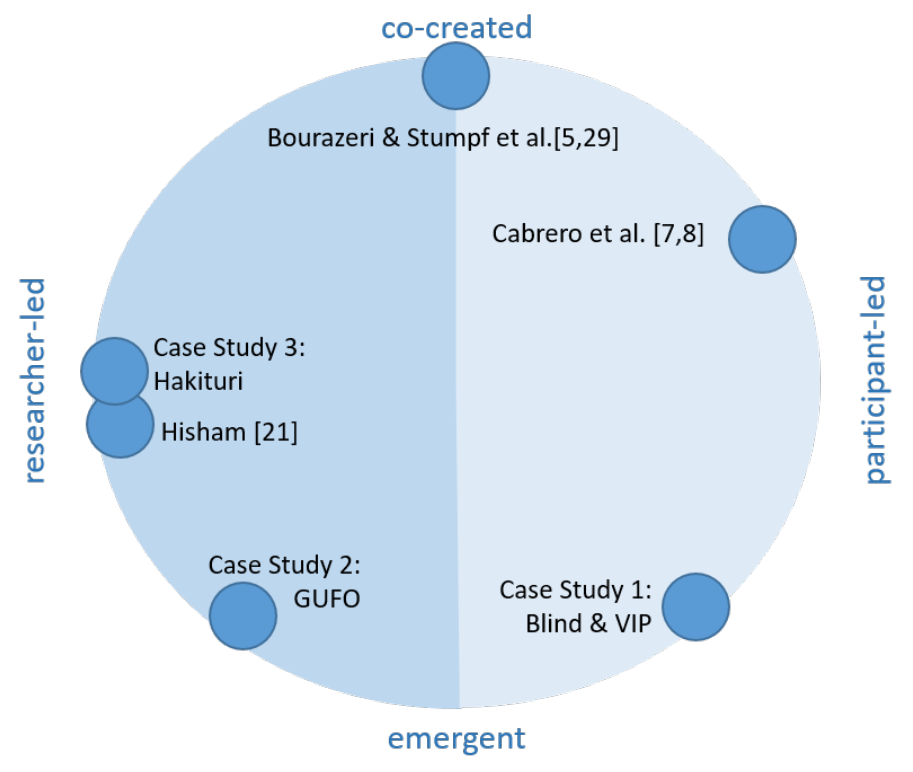

Figure 2: Comparison of Persona creation: researcher-led vs participant-led, co-created vs emergent

\subsection{Personas in the Design Process}

Figure 3 summarises the use of personas in the three case studies and places them in context with the phases of the technology design process (shown in blue). At the start of the design process, persona creation may happen as part of the technology design process $([11,12]$, Case Studies $1 \& 2)$, or outside of the process based on prior activities or context, such as experience of the researcher [37], visions and ideas for new concepts [21, 33, 43], and based on pre-existing data (Case Study 3). These prior activities outside of the technology design process are indicated by differently-coloured blocks in Figure 3. Furthermore, one can see that depending on the community, personas may be used throughout the complete technology design process, or rather as a clarification of concepts during the early phases. We can also consider how the initial personas that have been created can be extended with more details based on comments and input from the participants. For example, in the Hakituri project one of the older participant's primary concerns with using technology in the workplace was that it would lead to workers being replaced by machines, and therefore mean there was no future for his children and grandchildren in the only remaining industry in his local area. We added these concerns to the persona of Tahuri (a 52 year old supervisor) and as we continue to use the personas throughout the design process we can incorporate these actual concerns and other ideas through these extended personas.

\subsection{Use of Personas}

While in each of the case studies described personas were created and used in different ways, all were used within participatory design contexts as a way of eliciting more information from actual participants. In this way the personas can be considered as an enhancement to the participatory activities rather than as a replacement or representative for real users as is typically the case when personas are used with designers and other stakeholders early in the design process.

Using personas with potential or actual users within participatory design may be effective for a number of different reasons: it may help focus participants on particular needs or issues that they do not or would not identify in themselves; it may enable sharing of personal feelings with less emphasis on themselves by allowing attribution of thoughts and feelings to others rather than having to own them; it may facilitate group discussions where the focus is on a particular person (the persona) which avoids creating tensions between the actual participants. However, in the Hakituri project we did observe that while the younger participant group accepted the persona and were able to include them in their discussions, it did not necessarily lead to them providing more personal information. It did however provide some insights into how they felt things like fatigue-management were perceived within their group.

In general, we believe that given the results we have seen across the three case studies we can consider that using personas in this way is a useful tool for participatory and user-centred design. Also following their initial use, the personas can also be taken into the design space and used as necessary. For the participatory design process keeping the personas lightweight and 'low maintenance' means there is less obligation to use them or rely on them and this can be tailored within groups by the facilitator. However, keeping them authentic is also important.

\section{CONCLUSIONS AND FUTURE WORK}

In this paper we have discussed using personas in a different way than their typical use by technology designers. In our three case studies, we have used personas within participatory design so that 


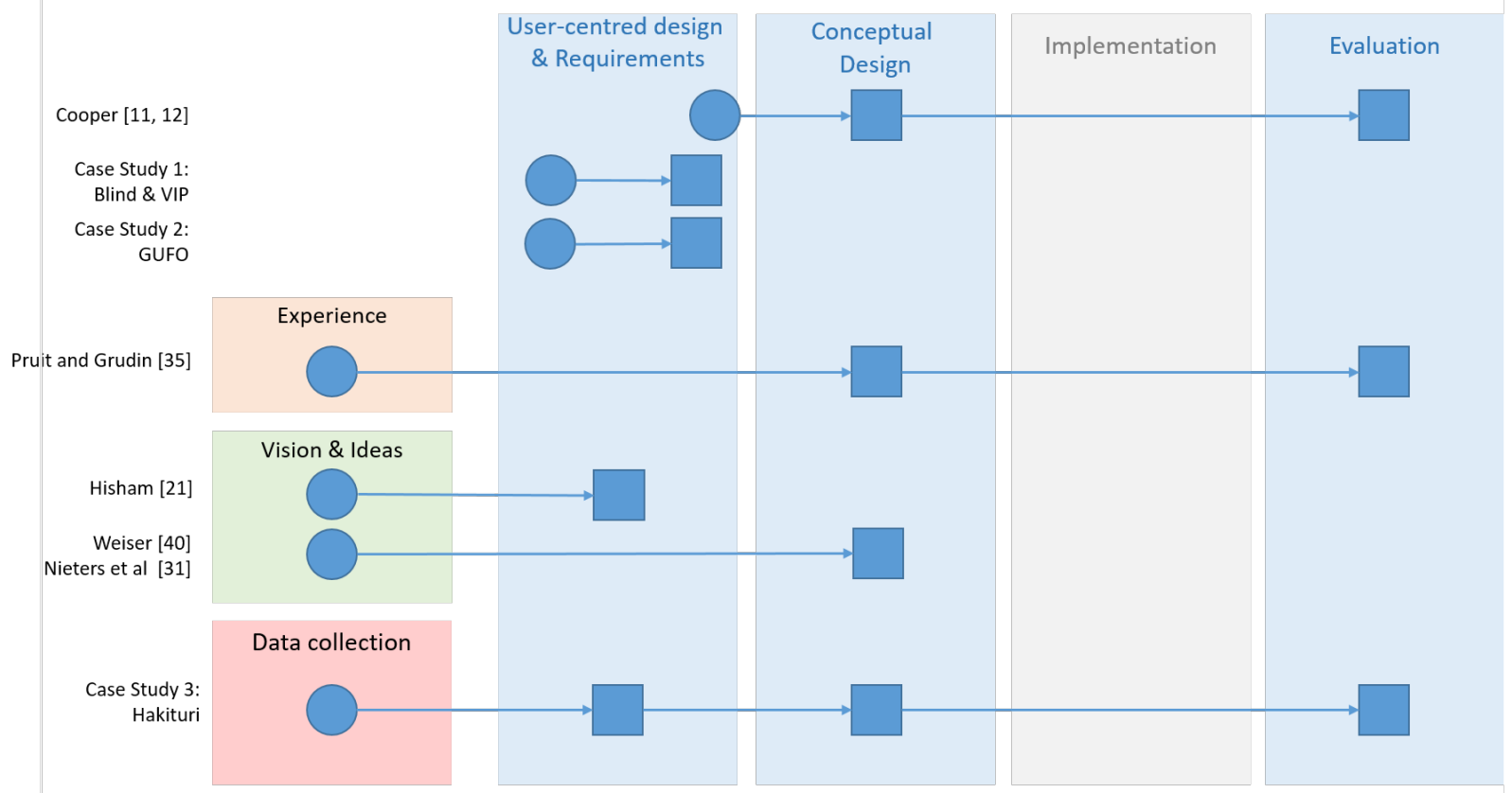

Figure 3: Comparison of Persona creation (circle) and use (square) in the technology design processes

they became a tool for the users rather than the designers. In the three case studies this approach was followed in different ways and we identified a number of benefits produced. We have also described potential problems with the approach and considered how these might be mitigated in future.

As part of future work, we are currently running a comparative study where two groups of participants are taking part in a design project based around mental health issues. One group will include the use of personas while the other will not. The goal is to see if we can discern any obvious differences in the information shared by the participants between the two groups and if this can be attributed to the inclusion (or not) of the personas.

This paper provides insights that are valuable to technology designers. It offers another way of working with participants, transferring the known concept of personas from a tool that allows designers to describe users' characteristics, to a tool that supports participants to explore and reflect on the broader needs of their user groups.

\section{ACKNOWLEDGMENTS}

We would like to thank all the people who participated in our studies for their time and patience.

In New Zealand, we would like to thank our collaborators the Centre for Health. We would also like to thank Worksafe NZ (in particular Nic Jessop), Lloyd Logging, and Johnson Contracting. Part of this work was funded under MBIE Smart Ideas Project UOWX1806, New Zealand.

In the UK, we would like to thank our collaborators in the GUFO Project, Cadscan Ltd (particularly Dr. Alistair Buchanan, Josh Cowburn and Dr. Jonathan Roberts) and Cosmonio Ltd. (particularly Dr. Ioannis Katramados and Dr. Oliver Hamilton). The GUFO Project was part funded by the Innovate UK fund (Grant No. 132666) of the UK government.

\section{REFERENCES}

[1] Tamara Adlin and John Pruitt. 2006. The persona lifecycle: keeping people in mind throughout product design. San Francisco, CA: Morgan Kaufmann.

[2] Farshid Anvari, Deborah Richards, Michael Hitchens, Muhammad Ali Babar. 2015. Effectiveness of persona with personality traits on conceptual design. IEEE/ACM 37th International Conference on Software Engineering (ICSE 2015), Florence, Italy. IEEE.

[3] Janet K. Belsky. 1998. The psychology of aging: theory, research and interventions (3rd edition). Belmont, CA, USA: Wadsworth Publishing.

[4] Hugh Beyer and Karen Holtzblatt, 1997. Contextual Design: Defining CustomerCentered Systems. San Francisco: Morgan Kaufmann.

[5] Aikaterini Bourazeri and Simone Stumpf. 2018. Co-designing smart home technology with people with dementia or Parkinson's disease. Proceedings of NordiCHI 18. New York: ACM Press.

[6] Judy Bowen, Annika Hinze and Christopher Griffiths. 2019. Investigating realtime monitoring of fatigue indicators of New Zealand forestry workers. Accident Analysis and Prevention, 126, 122-141. https://doi.org/10.1016/j.aap.2017.12.010

[7] Daniel G. Cabrero. 2015. User-created persona: Namibian rural Otjiherero speakers. Proceedings of SIGDOC '15, Limerick, Ireland. New York: ACM Press.

[8] Daniel G. Cabrero, Heike Winschiers-Theophilus and José Abdelnour-Nocera. 2016. A Critique of Personas as representations of "the other" in Cross-Cultural Technology Design. Proceedings of AfriCHI'16, November 21-25, 2016, Nairobi, Kenya. New York: ACM Press.

[9] Yen-Ning Chang, Youn-Kyung Lim and Erik Stolterman. 2008. Personas: from theory to practices. Proceedings of NordiCHI 2008, Lund, Sweden. New York: ACM Press.

[10] Christopher N. Chapman and Russell P. Milham. 2006. The personas' new clothes: methodological and practical arguments against a popular method. Proceedings of the Human Factors and Ergonomics Society 50th Annual Meeting.

[11] Alan Cooper. 1999. The inmates are running the asylum. New York: Macmillan.

[12] Alan Cooper, Robert Reimann, David Cronin, Christopher Noessel. 2014. About face: the essentials of interaction design (4th edition). Indianapolis, IN: Wiley.

[13] J.P. Djajadiningrat, William W. Gaver and J.W. Frens. 2000. Interaction relabelling and extreme characters: methods for exploring aesthetic interactions. Proceedings of Designing Interactive Systems (DIS '00). New York: ACM Press.

[14] Forestry Owners Association and Ministry for Primary Industries NZ, Facts and Figures 2016/2017. http://www.nzfoa.org.nz/resources/publications/facts-and-Figures, 2018 
[15] Shamal Faily and Ivan Flechais. 2010. Barry is not the weakest link: eliciting secure system requirements with personas. Proceedings of the 24th British HCI Group Annual Conference on People and Computers (BCS-HCI '10). British Computer Society.

[16] Shamal Faily and Ivan Flechais. 2011. Persona cases: a technique for grounding personas. Proceedings of CHI 2011. Vancouver, BC, Canada. New York: ACM Press.

[17] Blaithin Gallagher, D. Goswami, B. Ruff, Alastair Buchanan, J. Dura Rodriguez and Helen Petrie. 2011. Navigational support for people with vision impairment Proceedings of 10th International Conference on Low Vision. International Society for Low Vision Research and Rehabilitation. Kuala Lumpur.

[18] Christopher Griffiths. 2018. Investigating the use of wearable technology to support safety in the workplace (Thesis, Master of Philosophy (MPhil)). The University of Waikato, Hamilton, New Zealand. Retrieved from https://hdl.handle.net/10289/ 11981

[19] Christopher Griffiths, Judy Bowen and Annika Hinze. 2017. Investigating Wearable Technology for Fatigue Identification in the Workplace. In Proceedings of Human-Computer Interaction - INTERACT 2017 (Vol. 10514, pp. 370-380). Springer

[20] Jonathan Grudin and John Pruitt. 2002. Personas, participatory design and product development: an infrastructure for engagement. Proceedings of the Participatory Design Conference, Malmo, Sweden. pp $144-162$.

[21] Syariffanor Hisham. 2009. Experimenting with the use of persona in a focus group discussion with older adults in Malaysia. Proceedings of OzCHI '09: Proceedings of the 21st Annual Australian Computer-Human Interaction Special Interest Group. pp 333 - 336. New York: ACM Press.

[22] Valerie Johnson and Helen Petrie. 1998. Travelling safely: the problems and concerns of blind pedestrians. British fournal of Visual Impairment, 16(1), 27 - 31

[23] Soon-Gyo Jung, Jisun An, Haewoon Kawk, Moeed Ahmad, Lene Nielsen and Bernard J. Jansen. 2017. Persona generation from aggregated social media data Proceedings of CHI '17.New York: ACM Press.

[24] Soon-Gyo Jung, Joni Salminen, Haewoon Kawk, Jisun An, and Bernard J. Jansen 2018. Automatic persona generation (APG): a rationale and demonstration. Proceedings of CHIIR '18. New York: ACM Press.

[25] Frank Long. 2009. Real or imaginary: the effectiveness of using personas in product design. Irish Ergonomics Review, Proceedings of the IES Conference, Dublin.

[26] Jennifer McGinn and Nalini Kotamraju. 2008. Data-driven persona development Proceedings of $\mathrm{CHI}$ ' 08 . New York: ACM Press.

[27] Nicola Marsden and Maren Haag. 2016. Stereotypes and politics: reflections on personas. Proceedings of CHI '16, San Jose, CA, USA. New York: ACM Press.

[28] Tara Matthews, Tejinder K. Judge and Steve Whittaker 2012. How do designers and user experience professionals actually perceive and use personas? Proceedings of CHI '12. New York: ACM Press.

[29] Timothy Neate, Aikaterini Bourazeri, Abi Roper, Simone Stumpf and Stephanie Wilson. 2019. Co-created personas: engaging and empowering users with diverse needs within the design process. Proceedings of CHI '19. New York: ACM Press.

[30] Lene Nielsen. 2012. Acting as someone like me - personas in participatory innovation. In Proceedings of the Participatory Innovation Conference 2012, Melbourne,
Australia.

[31] Lene Nielsen, Soon-Gyo Jung, Jisun An, Joni Salminen, Haewoon Kwak and Bernard J. Jansen. 2017. Who Are Your Users?: Comparing Media Professionals' Preconception of Users to Data-driven Personas. Proceedings of the 29th Australian Conference on Computer-Human Interaction (OzChi '17), 602-606.

[32] Lene Nielsen and Kira Storgaard Hansen. 2014. Personas is applicable - a study on the use of personas in Denmark. Proceedings of CHI '14. New York: ACM Press.

[33] James E. Nieters, Subbarao Ivaturi and Iiftikhar Ahmed. 2007. Making personas memorable. Proceedings of CHI 2007. New York: ACM Press.

[34] Helen Petrie, Valerie Johnson, Thomas Strothotte, Rainer Michel, Andreas Raab, Lars Reichert, and A. Schalt. 1997. User-centred design in the development of a navigational aid for blind travellers. Human-Computer Interaction: INTERACT '97. (IFP TC13 International Conference on Human-Computer Interaction). London: Chapman and Hall.

[35] Helen Petrie, Christoph Schlieder, Paul Blenkhorn, Gareth Evans, Alasdair King, Anne-Marie O’Neill, George T. Ioannidis, Blaithin Gallagher, David Crombie, Rolf Mager, and Maurizio Alafaci . 2002. TeDUB: a system for presenting and exploring technical drawings for blind people. In K. Miesenberger, J. Klaus and W. Zagler (Eds.), Lecture Notes in Computer Science 239: Computers Helping People with Special Needs. Heidelberg: Springer Verlag.

[36] Christopher Power, Andree Freire, Helen Petrie and David Swallow. 2012. Guidelines are only half the story: accessibility problems encountered by blind users on the web. Proceedings of CHI 2012. New York: ACM Press.

[37] John Pruitt and Jonathan Grudin. 2003. Personas: Practice and theory. DUX '03: Proceedings of the 2003 Conference on Designing for User Experience. San Francisco, CA. pp 1 - 15 .

[38] Rebecca M. Quintana, Stephanie R. Haley, Adam Levick, Caitlin Holman, Ben Hayward and Mike Wojan. 2017. The persona party: using personas to design for learning at scale. CHI ' 17 Extended Abstracts, Denver, CO, USA. New York: ACM Press.

[39] Joni Salminen, Haewoon Kwak, Joao M. Santos, Soon-Gyo Jung, Jisun An and Bernard J. Jansen. 2018. Persona Perception Scale: developing and validating an instrument for human-like representations of data. CHI "18 Extended Abstracts. Montreal, QC, Canada. New York: ACM Press.

[40] Joni Salminen, Lene Nielsen, Soon-Gyo Jung, Jisun An, Haewoon Kwak and Bernard J. Jansen. 2018. "Is More Better?": Impact of Multiple Photos on Perception of Persona Profiles. Proceedings of ACM CHI Conference on Human Factors in Computing Systems (CHI2018). New York: ACM Press.

[41] Andreas Savva, Helen Petrie and Christopher Power. 2016. Types of problems elicited by verbal protocols for blind and sighted participants. In Proceedings of the 15th International Conference on Computers Helping People with Special Needs (ICCHP 2016).

[42] Thomas Strothotte, Valerie Johnson, Helen Petrie and Graeme Douglas. 1998. Evaluation of an orientation and navigation aid for visually impaired travellers. In I. Placencia-Porrero and E. Ballabio (Eds.), Improving the quality of life for the European citizen. IOS Press.

[43] Mark Weiser. 1991. The computer for the 21st century. Scientific American, 94-104. 